\title{
Interdisciplinary Analysis of Digital Library
}

\author{
Jiamei Yang*, Mengxia Cheng \\ School of Economics and Management, Nanjing University of Science and Technology, Nanjing, China \\ *Corresponding author: jokery77@163.com
}

Keywords: digital library, interdisciplinary research, bibliometrics, word frequency analysis.

\begin{abstract}
As a new discipline in the field of library science, the digital library has obvious interdisciplinary phenomenon. Studying its interdisciplinary trend and research topics will help scholars understand the development of the discipline and provide guidance for scholars to conduct interdisciplinary research. This paper studies the interdisciplinary situation of digital library from the perspectives of scholars' interdisciplinary publications and text content, revealing the relationship between digital library and other areas and the evolution of interdisciplinary research in digital library. Finally, the use of keyword frequency analysis reveals the themes of scholars' interdisciplinary research.
\end{abstract}

\section{Introduction}

Looking back on the development process of modern science, the discovery and solution of many major problems often involve the mutual exchange and mutual penetration of multiple disciplines. The intersection and integration of different disciplines can often give birth to new disciplines and new scientific frontiers, and it is most likely to produce major scientific breakthroughs. Therefore, interdisciplinary research is conducive to innovation has become a consensus among scholars. Some scholars have long been aware of the trend of interdisciplinary research in the field of library science, and believe that the development of interdisciplinary research in library science is the need for the development of library science itself. Digital Library (DL) is a new subject in the field of library science, and its interdisciplinary research phenomenon is remarkable.

\section{Literature Review}

Woodworth first proposed the term "Interdisciplinary". He believes that "interdisciplinary" is a research activity involving two or more disciplines that transcends the boundaries of a known discipline ${ }^{[1]}$. Since then, many scholars have put forward their own definitions of interdisciplinary research ${ }^{[2]}$. Porter et al. ${ }^{[3]}$ pointed out that there is no unified definition of "interdisciplinary research" at present, and scholars have different concepts about interdisciplinary, but they all emphasize that interdisciplinary research is no longer limited to a single discipline. Instead, multiple disciplines work together to solve complex real-world problems.

The research objects of interdisciplinary research are mainly academic papers. By dividing different parts of academic papers, interdisciplinary research can be divided into three perspectives: co-author perspective, citation perspective, and text perspective. Interdisciplinary research from the perspective of co-authoring refers to the research of interdisciplinary activities and laws through the perspective of the collaborator's information of the academic paper. Existing research includes interdisciplinary research from the information of the collaborators ${ }^{[4]}$, interdisciplinary research from the information of the collaborators' institutions ${ }^{[5]}$, and interdisciplinary research from the interdisciplinary team ${ }^{[6]}$. Interdisciplinary research conducted from the perspective of co-authors mostly explores the relationship between scientific cooperation and the output of interdisciplinary research results in an interdisciplinary context. At present, the most important research object of interdisciplinary research is the references of papers, that is, starting from the perspective of citations. Part of the research is mainly in the aspect of interdisciplinary law research ${ }^{[7]}$, and the research in the aspect of interdisciplinary knowledge transfer and knowledge point discovery ${ }^{[8]}$. Interdisciplinary 
research from the perspective of citations mainly studies interdisciplinary laws, interdisciplinary knowledge transfer, interdisciplinary knowledge point discovery and cross-topic identification. The interdisciplinary analysis of the text perspective is mainly through the statistical analysis of keywords, or the term extraction of the citation content in the text, excavating the content of the text, discovering and predicting interdisciplinary knowledge, and exploring the structure of subject knowledge and the relationship of subject knowledge. Interdisciplinary research under the textual perspective is mostly used to discover the hotspots and cross-topics of interdisciplinary research ${ }^{[9]}$.

Porter et al. ${ }^{[3]}$ believe that the common feature of interdisciplinary research is reflected in the transfer of information between disciplines. Pierce ${ }^{[1]}$ summarized the information transfer between disciplines into three types, namely, references from different disciplines, co-authors from scholars in different disciplines, and publication of literatures in interdisciplinary publications. Although previous studies made full use of citation analysis methods and co-authored analysis methods, from a single subject interdisciplinary situation study to a multi-disciplinary interdisciplinary situation comparative study, the third method of information transfer proposed by Pierce has not attracted attention. In the current era of big science, in addition to publishing papers in this subject, a scholar often publishes papers in several other disciplines. This is determined by the inherent unity of science. This paper combines scholars' interdisciplinary publications and textual perspectives, through quantitative analysis of all the literatures published by authors and related data in the digital library included in CNKI, to explore the characteristics and laws of interdisciplinary research in digital library. In order to have a clearer understanding of the interdisciplinary research situation of digital library, and provide certain reference for scholars to conduct interdisciplinary research.

\section{Data and Research Method}

China National Knowledge Infrastructure (CNKI) is currently the world's largest continuously dynamically updated full-text database of Chinese journals, and its source coverage has reached 99\%. Therefore, the analysis results obtained for interdisciplinary research of digital library are reliable. All the literatures included in the "China Academic Literature Network Publishing General Library" are divided into 168 subject digital library and more than 3000 sub-specialized digital library according to the "Chinese Book Classification". The digital library of each discipline is the general library of academic literature of the discipline. The data source of this literature comes from the "Digital Library" subject professional library.

Scholars refer to those who pursue learning, that is, those who specialize in academic research. In order to distinguish from general paper authors, we define the authors whose first author has published more than two literatures and published more than 2 literatures in core journals as scholars. Searching in the discipline library of CNKI, there are 3,659 interdisciplinary research scholars in the digital library. The data such as the units, research areas, core journal papers, first author papers, and total citation frequency of these scholars are collected and compiled. On the basis of these data, conduct an interdisciplinary analysis of digital library from the perspective of scholars' interdisciplinary publications. In addition, through the retrieval of 19,358 literatures in the "Digital Library" subject library(as of December 31, 2019), the analysis of the subject field and the keyword analysis of the literatures in different years are carried out to study the interdisciplinary research trend and research theme of the digital library from the text perspective.

\section{Interdisciplinary Analysis from the Perspective of Interdisciplinary Publishing}

The perspective of interdisciplinary publishing is different from the traditional citation perspective and co-author perspective. This perspective is to study the interdisciplinary situation of digital library from the interdisciplinary degree of scholars' papers and the field of interdisciplinary research by scholars themselves. It is reflected in the following two aspects, one is to show the degree of interdisciplinary research by digital library scholars through specialization indicators, and the other is to statistically analyze the specific subject areas of digital library scholars' interdisciplinary research, so as to show the relationship between digital library disciplines and these areas. 


\subsection{Quantitative measurement of scholars' interdisciplinary research degree}

In 2007, Porter et al. proposed the Specialization (S) indicator, which is used to measure the degree of diversification of the subjects published by authors. The formula for calculating the $S$ is shown in equation (1).

$$
S=\frac{\sum\left(P_{s C_{1}}^{2}+\ldots+P_{s C_{n}}^{2}\right)}{\sum\left(P_{S C_{1}}+\ldots+P_{S C_{n}}\right)^{2}}
$$

$P_{S C_{n}}$ refers to the number of literatures belonging to subject $n$, and $S \in[0,1]$. The higher the interdisciplinary nature of the scholar, the closer the $S$ value is to 0 .

In order to avoid the problem of duplicate names of authors, the search is mainly based on the author's name and the author's unit, and then the search results are manually checked to remove non-retrieving author's records. According to the search results, the professional degree of scholars can be calculated using equation (1), and the results are shown in Table 1(Top 10 by citations).

Table.1. Specialization of interdisciplinary digital library scholars

\begin{tabular}{|c|c|c|c|}
\hline Cited ranking & Specialization ranking & Name & Specialization \\
\hline 1 & 5 & Junping Qiu & 0.250 \\
\hline 2 & 10 & Bingsi Fan & 0.628 \\
\hline 3 & 2 & Qinghua Zhu & 0.165 \\
\hline 4 & 1 & Haiqun Ma & 0.136 \\
\hline 5 & 9 & Ping Ke & 0.600 \\
\hline 6 & 8 & Xiaolin Zhang & 0.370 \\
\hline 7 & 4 & Guoxin Li & 0.246 \\
\hline 8 & 3 & Xinning Su & 0.213 \\
\hline 9 & 6 & Jianming Zheng & 0.253 \\
\hline 10 & 7 & Xiaobin Huang & 0.326 \\
\hline
\end{tabular}

From Table 1, it can be seen that Haiqun Ma has the highest degree of interdisciplinary research, with a specialization of 0.136 , ranking first. Through CNKI's search results, it can be known that Ma Haiqun's research involves 30 research areas, and the number of literatures published in many areas exceeds 20, which shows that Haiqun Ma's high interdisciplinary nature. In addition, by comparing and observing the citation rankings and specialization rankings, it can be found that the total citations are not necessarily related to the degree of interdisciplinary research. Although many scholars such as Xinning $\mathrm{Su}(8 / 3)$ are ranked low by citations, they are highly interdisciplinary. Scholars such as Bingsi Fan(2/10) have more citations, but the degree of interdisciplinary research is low.

\subsection{Frequency analysis of subject areas of interdisciplinary research}

What is the mainstream subject area of interdisciplinary research by digital library scholars? This paper mainly examines the second and third research areas of digital library scholars. The frequency of the second subject area and the third subject area of interdisciplinary research is counted, and the corresponding frequency distribution is obtained. According to statistics, the second subject area of interdisciplinary research by digital library scholars involves 124 subject areas, and the third subject area involves 145 subject areas. Table 2 shows the frequency distribution of the second subject area (top 10) and the third subject area (top 10) of the interdisciplinary research by digital library scholars.

It can be seen from Table 2 that there is not much difference between the second subject area and the third subject area in the top 10 interdisciplinary research frequency of digital library scholars, with an overlap rate of $80 \%$, and only some areas have changed their rankings. Among them, computer software and computer applications, news and media, and higher education rank the top three in the second and third subject areas, indicating that computer software and computer applications, news and media, higher education and are closely linked with digital library. 
Table.2. Frequency distribution of subject areas for interdisciplinary research

\begin{tabular}{|c|c|c|c|c|}
\hline Rank & The second subject area & Freq & The third subject area & Freq \\
\hline 1 & $\begin{array}{c}\text { Computer Software and Computer } \\
\text { Applications }\end{array}$ & 2167 & $\begin{array}{c}\text { Computer Software and Computer } \\
\text { Applications }\end{array}$ & 562 \\
\hline 2 & News and Media & 175 & News and Media & 348 \\
\hline 3 & Higher Education & 144 & Higher Education & 235 \\
\hline 4 & Publishing & 133 & Internet Technology & 202 \\
\hline 5 & Civil and Commercial Law & 98 & Publishing & 194 \\
\hline 6 & Internet Technology & 65 & Civil and Commercial Law & 136 \\
\hline 7 & Business Economy & 58 & Scientific Research Management & 91 \\
\hline 8 & $\begin{array}{c}\text { Medical Education and Medical } \\
\text { Marginal Disciplines }\end{array}$ & 56 & $\begin{array}{c}\text { Educational Theory and Educational } \\
\text { Management }\end{array}$ & 85 \\
\hline 9 & Scientific Research Management & 38 & Business Economy & 85 \\
\hline 10 & Light Industry Handicraft Industry & 31 & Culture & 71 \\
\hline
\end{tabular}

\section{Interdisciplinary Analysis from the Perspective of Text}

This chapter mainly studies the interdisciplinary situation of digital library from the traditional textual perspective. The subject area of the literature and the keywords of the literature can reflect the text content of the literature to a certain extent. Therefore, this section is mainly divided into two aspects, one is to study the evolution process of digital library interdisciplinary research based on the distribution of digital library literatures and the time factor, and the second is to use the keywords of the literature to study the key themes of the interdisciplinary research of the digital library.

\subsection{The evolution process of digital library interdisciplinary research}

This section considers the time dimension and examines the distribution of digital library literatures in different time periods, so as to reflect the evolution of digital library interdisciplinary research. In the "Digital Library" subject library, search the literatures of the digital library and analyze the subject areas of the literatures in different years.

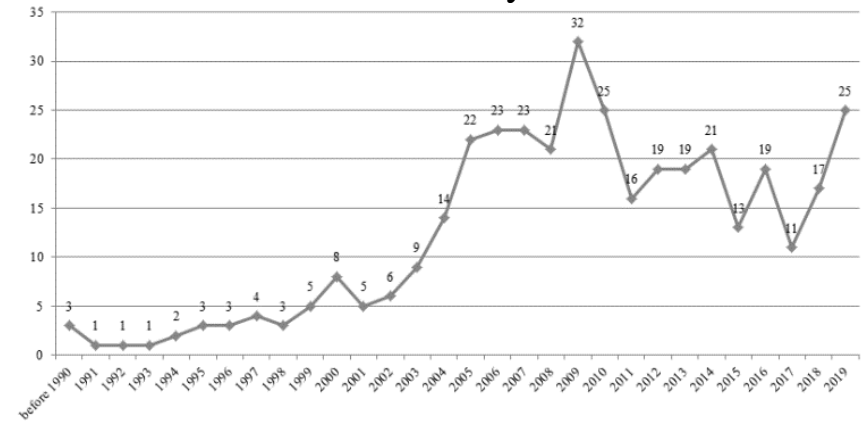

Figure.1. Trends in the number of disciplines in interdisciplinary research in digital library

In the "digital library" subject library, the literatures of the digital library are retrieved by year, and the subject areas of the literatures are counted. The result is shown in Figure 1. It can be seen from the figure 1 that before 2000, the literature in the digital library field is not closely related to other disciplines, and the number of disciplines in interdisciplinary research is small without much change. After 2000, the number of disciplines in interdisciplinary research in digital library gradually increased, indicating that the literature in the field of digital library intersects with more and more subject areas, and reached a peak of 32 in 2009. After that, the number of disciplines in interdisciplinary research in digital library fluctuated around 18. It can be seen that with the development of the digital library field, cooperation with other subjects and practical applications have gradually increased.

In order to understand the evolution of interdisciplinary research in the field of digital library, we 
take computer software and computer application disciplines with the highest frequency in interdisciplinary fields as examples to study the evolution of interdisciplinary research content.

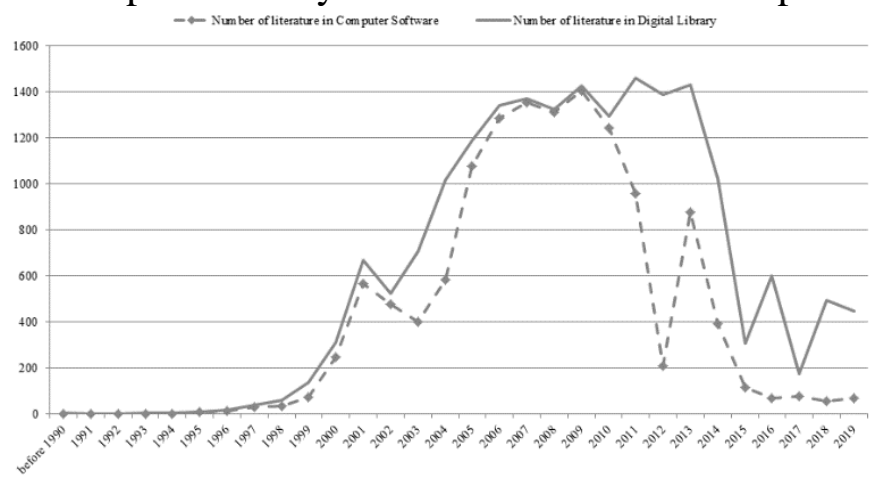

Figure 2. The evolution of interdisciplinary research on the computer software

It can be clearly seen from Figure 2 that the two trends are basically the same, and since the beginning of the digital library, it has already crossed with computer software and computer application disciplines. The digital library is a broadband multimedia network and massive information management system. The collection and processing, maintenance, management and storage of digital information resources in digital library are closely related to the development of technology. Therefore, it is foreseeable that the digital library, computer software and computer application will produce subject intersection. And with the passage of time, with the advancement of technology, the literature in the field of interdisciplinary computer software and computer applications has gradually increased, and the trend of change is basically in line with the number of literatures in the digital library.

\subsection{Analysis of interdisciplinary research topics in digital library}

According to the previous research results, we can find that computer software and computer applications are closely related to digital library. In order to further study the specific content of interdisciplinary research, this section selects literatures with a total citation greater than or equal to 15 to analyze the frequency of keywords to further analyze the themes of interdisciplinary research by digital library scholars, and provide guidance for digital library scholars in interdisciplinary research. We finally obtains 1,075 effective keywords. Table 3 lists the top 20 keywords in terms of frequency.

Table.3. Keywords for interdisciplinary research on computer software and computer applications

\begin{tabular}{|c|c|c|}
\hline Rank & Keyword & Freq \\
\hline 1 & Digital library & 727 \\
\hline 2 & Personalized service & 81 \\
\hline 3 & Mobile library & 51 \\
\hline 4 & Library & 49 \\
\hline 5 & Information service & 47 \\
\hline 6 & Cloud computing & 37 \\
\hline 7 & Digital resources & 32 \\
\hline 8 & Electronic reading room & 32 \\
\hline 9 & University library & 29 \\
\hline 10 & Metadata & 27 \\
\hline
\end{tabular}

It can be seen from Table 3 that in the cross-research of digital library field and computer software and computer application field, the keyword "digital library" has the highest frequency of 727 (accounting for $72.2 \%$ of the total number of literatures), which is consistent with the literature belonging to the field of digital library. Followed by personalized services, mobile library, information service, cloud computing, etc.

The frequency of "personalized service" is 81, ranking second. The development of computer 
technology, the improvement of algorithms, and the development of various personalized recommendation systems have made it easier to analyze user behavior, and provided strong support to meet the needs of different users and improve the quality of digital library services. The frequency of "Mobile Library" is 51, ranking third. With the popularization of mobile terminal devices such as smart phones and IPads, the functions of digital library have been further expanded in the wireless mobile network environment, and mobile libraries have become an extension and supplement of digital library electronic information services. The development of mobile libraries is also inseparable from the support of computer technology.

\section{Conclusions}

This paper combines the perspectives of scholars' interdisciplinary publishing and the perspective of text content to explore the trend of interdisciplinary research in digital library. It mainly reveals the influence of computer software and computer application fields on the subject of digital library, as well as the themes of interdisciplinary research, and provides guidance for scholars to conduct interdisciplinary research in a targeted manner. However, this paper also has some shortcomings, and the research can be further deepened. This paper has deeply studied the cross-research relationship between digital library and computer software and computer application fields, and we can study the relationship between more fields and digital library in the future. The time dimension can be added to the analysis of the research topics of digital library across different fields to explore the theme evolution process and trends of digital library in different cross-disciplinary research fields, and provide ideas and directions for scholars in interdisciplinary research.

\section{References}

[1] Liu Z L. Interdisciplinary research in the era of interdisciplinary science[J]. Studies in Science of Science, 1993(2): 11-18+4. (in Chinese)

[2] Angelstam P, Andersson K, Annerstedt M, et al. Solving Problems in Social-Ecological Systems: Definition, Practice and Barriers of Transdisciplinary Research [J]. Ambio, 2013, 42(2):254-265.

[3] Porter A L, Roessner D J, Heberger A E. How interdisciplinary is a given body of research? [J]. Research Evaluation, 2008, 17(4):273-282.

[4] Abramo G, D’Angelo, Ciriaco Andrea, et al. Do interdisciplinary research teams deliver higher gains to science? [J]. Scientometrics, 2017, 111(1):317-336.

[5] Wang W, Wu Y, Pan Y. An investigation of collaborations between top Chinese universities: a new quantitative approach [J]. Scientometrics, 2014, 98(2):1535-1545.

[6] Melgarejo R, Cadena P. A Proposal Model to Monitor Interdisciplinary Research Projects in Latin American Universities [J]. IEEE Revista Iberoamericana de Tecnologias del Aprendizaje, 2015, 10(3):1-1.

[7] Noorden R V. Interdisciplinary research by the numbers [J]. Nature, 2015, 525(7569): 306-307.

[8] Zhu Y, Yan E. Dynamic subfield analysis of disciplines: an examination of the trading impact and knowledge diffusion patterns of computer science[J]. Scientometrics, 2015, 104(1):335-359.

[9] Chua A Y K, Yang C C. The Shift Towards Multi-Disciplinarity in Information Science[J]. Journal of the American Society for Information Science and Technology, 2008, 59(13):2156-2170. 